\title{
Compliant prosthetic wrists entail more natural use than stiff wrists during reaching, not (necessarily) during manipulation
}

Gunter Kanitz, Federico Montagnani, Marco Controzzi, Christian Cipriani,

(C) 2018 IEEE. Personal use of this material is permitted. Permission from IEEE must be obtained for all other uses, in any current or future media, including reprinting/republishing this material for advertising or promotional purposes, creating new collective works, for resale or redistribution to servers or lists, or reuse of any copyrighted component of this work in other works. The DOI associated with this publication is DOI: 10.1109/TNSRE.2018.2847565. 


\title{
Compliant prosthetic wrists entail more natural use than stiff wrists during reaching, not (necessarily) during manipulation
}

\author{
Gunter Kanitz, Federico Montagnani, Marco Controzzi, Member, IEEE, and \\ Christian Cipriani, Senior Member, IEEE
}

\begin{abstract}
Developing an artificial arm with functions equivalent to those of the human arm is one of the challenging goals of bioengineering. State of art prostheses lack several degrees of freedom and force the individuals to compensate for them by means of compensatory movements, which often result in residual limb pain and overuse syndromes. Passive wrists may reduce such compensatory actions, nonetheless to date their actual efficacy, associated to conventional myoelectric hands is a matter of debate. We hypothesized that a transradial prosthesis would allow simpler operation if its wrist behaved compliant during the reaching and grasping phase, and stiff during the holding and manipulation phase. To assess this we compared a stiff and a compliant wrist and evaluating the extent of compensatory movements in the trunk and shoulder, with unimpaired subjects wearing orthoses, while performing nine activities of daily living taken from the Southampton Hand Assessment Procedure. Our findings show indeed that the optimal compliance for a prosthetic wrist is specific to the phase of the motor task: the compliant wrist outperforms the stiff wrist during the reaching phase, while the stiff wrist exhibits more natural movements during the manipulation phase of heavy objects. Hence, this study invites rehabilitation engineers to develop wrists with switchable compliance.
\end{abstract}

Index Terms- Artificial limbs, Wrist, Compensatory movements, Prosthetics.

\section{Introduction}

$\mathrm{T}$ HE last decade has seen significant changes in the field of upper limb prosthetics. A deeper understanding of basic scientific questions in neurophysiology and neuroscience was mated by novel surgical techniques for the treatment of the patients and by new components such as articulated hands and

This work was partially supported by the European Commission under the DeTOP project (LEIT-ICT-24-2015, Grant no. 687905), and partially by INAIL (the Italian national workers' compensation) under the CECA 2020 project. The work of C. Cipriani was supported in part by the European Research Council under the MYKI project (ERC2015-StG, Grant no. 679820).

G. Kanitz, F. Montagnani, M. Controzzi and C. Cipriani, are with The BioRobotics Institute, Scuola Superiore Sant'Anna, V.le Piaggio 34, 56025 Pontedera (PI) Italy. (e-mail: ch.cipriani@santannapisa.it). myocontrollers that reached the markets. In particular myoelectric prostheses have gained increased dexterity, nonetheless the complexity and the functionality of the muscle-skeletal structure of the upper limb is far from being replicable. In fact even the most advanced hand prostheses represent trivial simplifications of the biological hand due to the lack of compact and powerful actuators [1]. This implies that only a reduced set of movements can be fitted inside the prosthesis, as clearly proved by current prosthetic wrist designs.

Modern prostheses usually include a simple wrist consisting of an active or passive rotator that enables the pronation/supination of the hand. Albeit simple it is known that this degree of freedom (DoF) contributes significantly to the unimpaired hand function and was shown valuable for amputees using it during activities of daily living (ADLs)[2][4]. Wrists with passive flexion/extension and/or radial/ulnar deviation that can be fitted in upper limb prostheses also exist [3], [5]-[8]. These wrists enhance the dexterity of the prosthesis and reduce the need by the individuals to compensate for the missing DoFs by means of unnatural movements of the elbow, shoulder and trunk [9]-[11]. The latter, namely compensatory movements, induce excessive stresses on the joints and the muscle-skeletal system, which cause discomfort and secondary injuries in the long run [12][14]. Passive wrists can be classified based on their compliance, i.e. stiff or compliant. Stiff wrists enable the user to manually orientate and lock the hand in a desired and firm position. Compliant wrists exhibit an elastic behavior and akin to the other ones can be manually locked in a certain position. As their name suggests compliant wrists allow for adaptation of the prosthesis during ADLs involving reaching and grasping.

The actual efficacy of passive wrists, associated to conventional 1 DoF myoelectric hands was poorly investigated so far. Kyberd compared the performance of different passive wrists as measured by the Southampton Hand Assessment Procedure (SHAP) [15]. He showed that a 2 DoFs compliant wrist outperformed the stiff wrists in all of the SHAP tasks with the exception of those where a power grasp was required [5]. The group of users surveyed by Petersen and colleagues reported functional improvements in a wide range of ADLs (in particular in bimanual tasks and in tool manipulation) while using 2 DoFs compliant wrists [6]. Nonetheless they also claimed that lifting heavy objects 


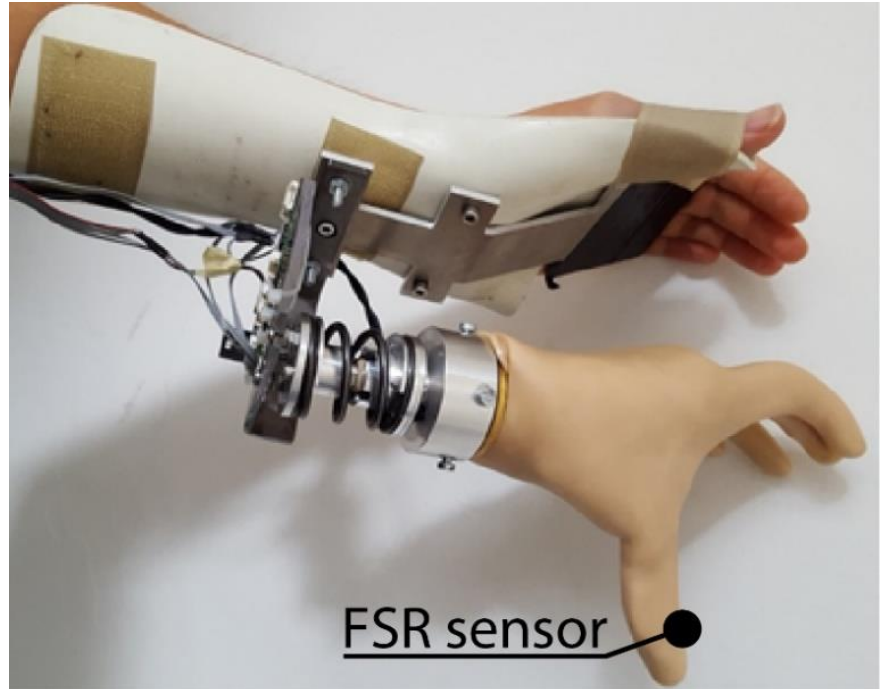

Fig. 1 Prosthetic hand/wrist emulator. Single degree of freedom prosthetic hand with wrist having two switchable degrees of compliance, mounted on an orthosis to be used by non-amputee subjects.

proved difficult and it was aided by locking the wrist. Recently Deijs and coworkers assessed compliant and stiff wrists used by amputees in their daily lives, in a two weeks study [7]. The subjects were repeatedly evaluated by means of the SHAP, a survey and a motion analysis test that measured the compensatory movements induced by the different wrists at the shoulder level. The authors concluded that neither the SHAP nor the motion analysis test showed significant differences among the different wrists. However, the subjects claimed to prefer the compliant wrist for most of the tasks, except for those where a stable grasp was required (in which the stiff wrist was preferred instead). All in all, the few studies conducted so far agree on task-specific advantages of compliant or stiff wrists, albeit they either remain inconclusive as they fail to show significant functional differences between wrists [7] or present rather anecdotal outcomes from open questionnaires [5], [6]. We argue that taken together, these studies suggest that during the reaching phase stiff wrists induce users to perform compensatory movements, whereas during the holding and manipulation phase of heavy objects, compliant wrists prove unpractical.

Based on these observations in this study we sought to isolate and analyze the different phases of the motor task using a prosthesis with a passive wrist, and to identify the most appropriate degree of compliance, in each phase. We hypothesized that a transradial prosthesis would allow easier and more natural operation if its wrist behaved compliant during the reaching and grasping phase, and stiff during the holding and manipulation phase. To test this we developed a mechanical wrist that could be switched to exhibit either a stiff or compliant behavior. We coupled the wrist with a conventional 1 DoF myoelectric hand onto an orthosis to be used by non-amputee subjects (Fig. 1), who volunteered in using it to perform ADLs, under different experimental conditions (stiff or compliant wrist). Taking the suggestion by Deijs and colleagues we measured the movements of the arm and also of the trunk while wearing the end-effector (emulated hand plus wrist) and compared them with a control configuration, namely the unimpaired and unconstrained hand/wrist. We used the movement deviations from the control condition (i.e. the compensatory movements) as the performance metric of the experimental configurations, under the assumption that more natural movements yielded to lower deviations [7], [16]-[18]. Our results, although acquired on unimpaired subjects wearing an emulator, prove that the optimal compliance for a prosthetic wrist is specific to the phase of the motor task. They also suggest that a wrist with two degrees of compliance could significantly reduce the compensatory movements required to operate the prosthesis, if switched - from compliant to stiff - in synchronous with the onset of the grasp.

\section{METHODS}

\section{A. Subjects and experimental procedure}

10 right-handed able-bodied subjects (10 males, aged 22-49 years old with a median of 31 years) free of any motor disorders participated to this study. Informed consent in accordance with the Declaration of Helsinki was obtained from each subject before conducting the experiments. The study was approved by the local ethical committee of the Scuola Superiore Sant'Anna, Pisa, Italy. The methods were carried out in accordance with the approved guidelines.

The subjects were asked to perform nine ADLs, chosen from the SHAP, in two experimental conditions wearing an experimental orthosis, and in the control condition with their unconstrained arm (Fig. 2). The nine ADLs covered a range of everyday activities and each primarily involved one of the main grips. The ADLs were: page turning, jar lid opening, jug pouring, carton pouring, full jar transport, empty tin transport, tray transport, using a key in a keyhole and door handle handling (Fig. 2). Two of the ADLs were bimanual (i.e. jar lid opening and tray transport; Fig. 2b and Fig. 2g). All required a precise orientation of the hand, i.e. precise movements at the shoulder and arm levels. With respect to the SHAP protocol we increased the weight of the carton of juice and of the jar by 500 grams as we deemed them too light to properly represent the spectrum of objects handled in the everyday life.

The experimental orthosis included a 1 DoF myoelectric hand (SensorHand - Otto Bock, Vienna, Austria) and a custom mechanical wrist that exhibited two switchable degrees of compliance: i.e. compliant or stiff (Fig. 1). The wrist was based on a ball joint embedding a locking pin; by manually locking/unlocking the pin it was possible to switch between compliant and stiff modes. In the compliant mode a compression spring constrained between two frames offered a compliance of $\sim 60 \mathrm{Nmm} /{ }^{\circ}$ (akin to commercial wrists, e.g. the Multiflex wrist by Touch Bionics). This grade was enough to keep the wrist practically aligned (unbent) when sustaining the hand alone, during slow movements. In the stiff mode the wrist was practically locked and aligned with the subject's forearm. In one experimental condition (stiff condition - SW) the wrist was kept in the stiff mode, while in the other condition (compliant condition - CW) it was maintained compliant. The orientation of the myoelectric hand in the orthosis (i.e. the wrist rotation angle) was fixed in a slight more pronated way with respect to the subject's wrist, in a way to ease grasping (Fig. 1). Such orientation was maintained 
across all subjects. When wearing the orthosis the subjects could control the opening/closing of the myoelectric hand by means of two myoelectric sensors (MyoBock 13E200, Otto Bock, Duderstadt, Germany) and a two-state amplitude modulated controller [19] implemented on a microcontroller, on a custom board. During the control condition (CC) the subjects performed the ADLs with their unconstrained arm (Fig. 2).

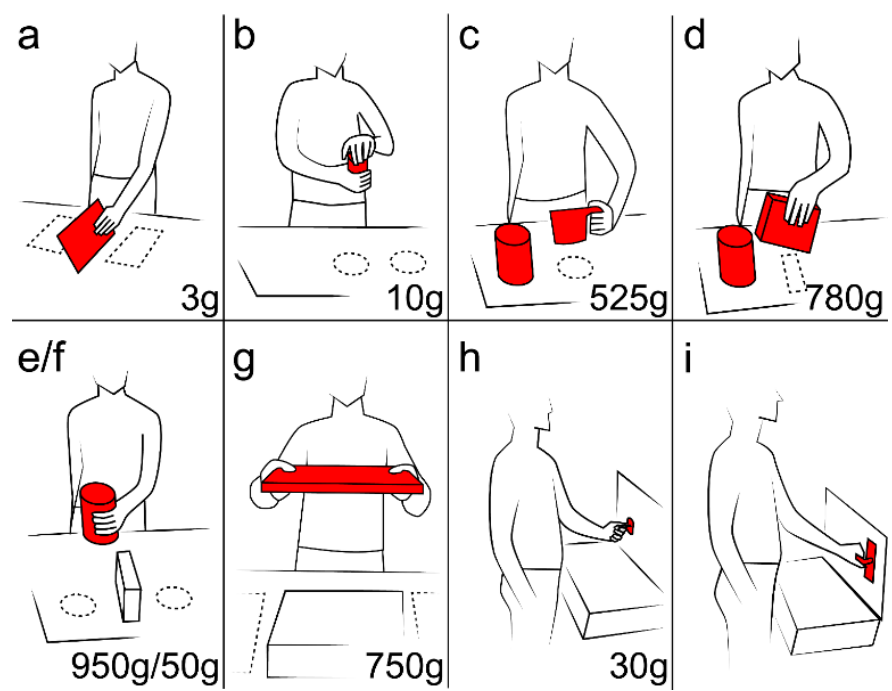

Fig. 2 Experimental tasks using a subset of the SHAP materials: (a)page turning, (b) jar lid opening, (c) jug pouring, (d) carton pouring, (e) full jar transport, (f) empty tin transport, (g) tray transport, (h) using a key in a keyhole, (i) door handle handling. In the lower part of the panel the weight of the manipulated object.

The two experimental conditions ( $\mathrm{SW}$ and $\mathrm{CW}$ ) were tested in an alternate order across subjects (i.e. five subjects started with $\mathrm{SW}$, the other five with $\mathrm{CW}$ ); the control condition was tested last in order to fit the orthosis once. The ADLs were always performed with the left arm (which proved the nondominant for all subjects) and with the subject standing in front of a table with the experimental materials. The beginning and end of each trial/recording were self-signaled by the subject by hitting a pushbutton. In particular, starting with the left arm in a home position, the subject had to: (i) hit the pushbutton with his/her right hand, (ii) perform the task with the left arm, (iii) return the left arm to the home position and (iv) hit the pushbutton again with his/her right hand. Notably, subjects were instructed to execute the tasks at a self-paced speed and as naturally as possible, rather than as fast as possible. The first time each new task was presented, the subject was taught/given hints on how to perform it with the orthosis and was left free to rehears until he/she was able to reliably perform it. The tasks were presented in the order of appearance of the SHAP and each one was deemed completed when five satisfactory repetitions were performed. Trials not deemed properly executed by the subject were discarded and repeated akin to the SHAP protocol [15].

Two inertial measurement units (IMU) (3-Space, Yost Labs, Portsmouth, $\mathrm{OH}$ ) and two linear potentiometers were used for recording the subjects' trunk, arm and wrist kinematics (sampling rate $100 \mathrm{~Hz}$ ). The IMUs were fixed on the trunk and left humerus in order to reconstruct, offline, the angular trajectories $(\alpha)$ and the compensatory movements of four crucial body segments, during the reaching and grasping phase. Indeed the chosen body segments were those that are typically engaged in compensatory movements with upper limb myoelectric prostheses [7], [16], [20], [21], , i.e.: the trunk lateral $\left(\mathrm{T}_{\mathrm{L}}\right)$, and forward $\left(\mathrm{T}_{\mathrm{F}}\right)$ movements (Fig. 3a-b), the glenohumeral abduction/adduction $\left(\mathrm{G}_{\mathrm{A} / \mathrm{A}}\right)$, and forwardflexion/backward-extension $\left(\mathrm{G}_{\mathrm{F} / \mathrm{E}}\right)$ (Fig. 3c-d). Notably as the forearm pronation/supination was allowed in all the

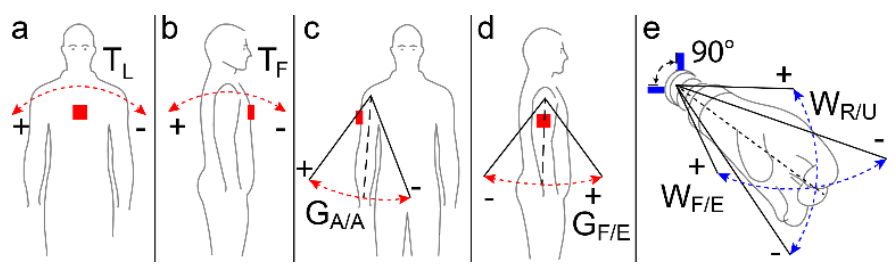

Fig. 3 Body segments measured and sensor placement (in red the inertial measurement unit; in blue the linear potentiometers).

conditions, the relevant compensatory movements were all at the level of the shoulder and the trunk [20], [22], [23]. The linear potentiometers, embedded in a custom brace (Fig. 3), were used to measure the wrist flexion/extension $\left(\mathrm{W}_{\mathrm{F} / \mathrm{E}}\right)$ and radial/ulnar deviation $\left(\mathrm{W}_{\mathrm{R} / \mathrm{U}}\right)$ (Fig. 3e), in order to assess the stability of the wrist during the holding and manipulation phase. Finally a force sensing resistor (FSR) mounted on the fingertip of the thumb was used to detect contact events and to segment the data offline.

Before the experimental conditions the orthosis was worn and the two EMG sensors were placed on the wrist flexors and extensors muscles. The EMG controller was then tuned in a way the subject could easily operate the hand at his/her will. Afterwards the wrist sensors were calibrated (once) and the actual experiment started. The IMU were calibrated before each recording (repetition) begun (at the time the subject hit the pushbutton) to prevent drift. After the $\mathrm{SW}$ and $\mathrm{CW}$ conditions the orthosis was removed and the wrist sensors recalibrated on the unconstrained hand (control condition - CC).

\section{B. Data analysis}

The start and end instants of each trial/recording were determined from the pushbutton signal. The first time derivative of the FSR data was computed and filtered (zero phase first order low-pass filter; cut-off frequency of $15 \mathrm{~Hz}$ ); the global maximum and minimum of this signal were thus used to identify the grasp and release instants of the ADL, respectively. The latter were used to segment each recording into three phases: i) reaching and grasping (RG), ii) holding and manipulation (HM), and iii) releasing and return to home (RR). The recordings were normalized in time prior to further processing.

The trajectories of the four body segment angles $(\alpha)$ during the experimental conditions (SW or $\mathrm{CW}$ ) were reconstructed for the entire duration of the recordings following the procedures described in previous studies [7], [24]. Their deviations $(\Delta)$ from the control condition trajectory (namely, the compensatory movements) were computed only when relevant, i.e. during the RG phase [20], [22]. During the HM phase the deviations of the wrist angles were computed, 
instead, in order to assess how close to the natural condition the artificial wrist behaved. The RR phase was no longer considered as it accounted for a part of the task of no interest for the present study.

For each angle $\alpha \in\left[\mathrm{T}_{\mathrm{L}}, \mathrm{T}_{\mathrm{F}}, \mathrm{G}_{\mathrm{A} / \mathrm{A}}, \mathrm{G}_{\mathrm{F} / \mathrm{E}}, \mathrm{W}_{\mathrm{F} / \mathrm{E}}, \mathrm{W}_{\mathrm{R} / \mathrm{U}}\right]$, wrist configuration $j \in[S W, C W]$, phase $p \in[R G, H M]$, and repetition $r \in[1, \ldots, 5]$, of task $t \in[1, \ldots, 9]$, the deviation from the control condition, in the normalized time $\mathrm{x}$, was computed as:

$$
\Delta_{\alpha}(j, p, t, r, x)=\frac{|\alpha(j, p, t, r, x)-\bar{\alpha}(p, t, x)|}{\operatorname{ROM}(\alpha)}
$$

where $\bar{\alpha}$ was the average $\alpha$ across the 5 repetitions in the control condition, and $\operatorname{ROM}(\alpha)$ was the average Range of Movement of $\alpha$, as indicated in physiology textbooks [26]. To simplify the analysis of the results the pairs of angles of the trunk $\left(\mathrm{T}_{\mathrm{L}}\right.$ and $\left.\mathrm{T}_{\mathrm{F}}\right)$, those of the shoulder $\left(\mathrm{G}_{\mathrm{A} / \mathrm{A}}, \mathrm{G}_{\mathrm{F} / \mathrm{E}}\right)$ and of the wrist $\left(\mathrm{W}_{\mathrm{R} / \mathrm{U}}, \mathrm{W}_{\mathrm{F} / \mathrm{E}}\right)$ were fused into a single variable $\left(\Delta_{\mathrm{B}}\right)$, thus obtaining three variables $\left(\Delta_{\mathrm{T}}, \Delta_{\mathrm{S}}\right.$ and $\left.\Delta_{\mathrm{W}}\right)$ :

$$
\begin{gathered}
\Delta_{B}(j, p, t, r, x)=\frac{1}{2} \sum_{i=1}^{2} \Delta_{\alpha, i}(j, p, t, r, x) \\
\text { where: } \alpha_{i}=\left\{\begin{array}{cc}
\alpha\left(T_{L}, T_{F}\right), & \text { if } B=T \rightarrow \text { Trunk } \\
\alpha\left(G_{A / A}, G_{F / E}\right), & \text { if } B=S \rightarrow \text { Shoulder } \\
\alpha\left(W_{R / U}, W_{F / E}\right), & \text { if } B=W \rightarrow \text { Wrist }
\end{array}\right.
\end{gathered}
$$

The mean deviation in time $\left(\overline{\Delta_{B}}\right)$ was finally computed and used as the performance metric; the $\overline{\Delta_{B}}$ induced by the two configurations were statistically compared using a Wilcoxon ranked sum test for each body segment and task $(\mathrm{p}=0.05)$.

\section{Results}

The duration of the whole experiment was about $1.5 \mathrm{~h}$ for each subject, including the setup/preparation time. The 10 subjects quickly learned how to perform the nine ADL tasks in a stereotypical manner in each of the three conditions (stiff wrist - SW, compliant wrist $-\mathrm{CW}$, control condition $-\mathrm{CC}$ ), as observed by the trajectories of the trunk, arm and wrist body segments (Fig. 4). In fact, for each subject and each task, the aggregated data from the five recordings demonstrated a median standard deviation lower than $13 \%$ of the ROM, across repetitions (Fig. 4). The inter-subject variability was also low with regards to the body segments movements (median standard error of mean lower than $9 \%$ of the ROM, across subjects). The movements proved highly stereotypical also in time, prior to normalization. The aggregated data from the five recordings demonstrated a median mean and standard deviation of the execution time of $8.7 \pm 0.7 \mathrm{~s}$ for the $\mathrm{SW}$ and $8.5 \pm 0.7 \mathrm{~s}$ for the $\mathrm{CW}(5.6 \pm 0.3 \mathrm{~s}$ for $\mathrm{CC})$. The average intersubject execution time variability was also low (standard error of mean lower than $8 \%$ of the average execution time, across subjects).

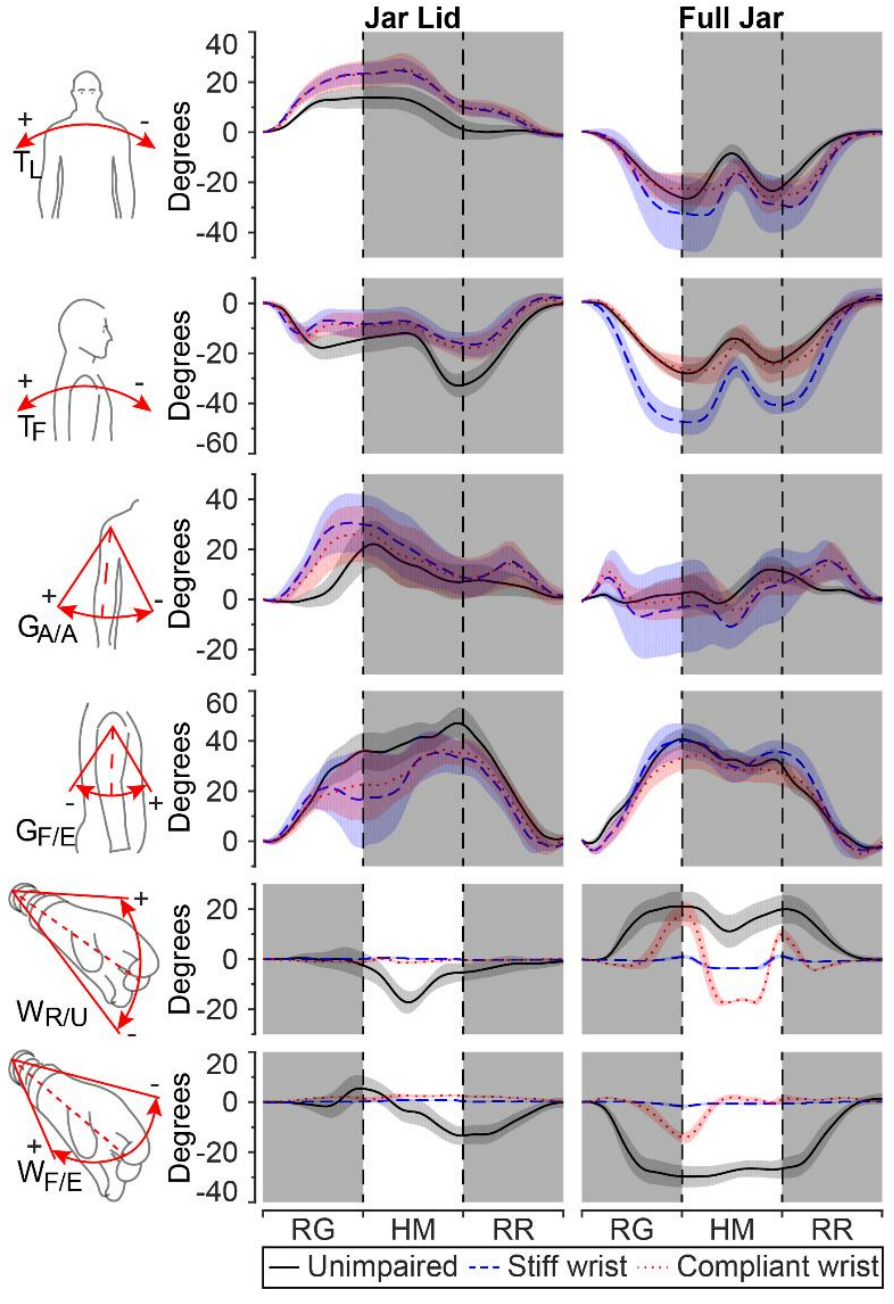

Fig. 4 Angular trajectories of the arm, trunk and wrist angles during the jar lid opening and the full jar transport tasks. For each condition (black bold = unimpaired; blue dashed $=$ stiff wrist; red dotted $=$ compliant wrist) the average trajectory across all subjects (solid line) and the 95\% confidence interval (shades) are shown for each phase $(R G=$ reaching and grasping; $\mathrm{HM}=$ holding and manipulation; $\mathrm{RR}=$ releasing and return to home). Phases were delimited using the FSR signal. The subtle movements of the wrist in the SW configuration were due to a small backlash in the mechanism.

When using the $\mathrm{CW}$ for grasping an object, the subjects learned that they could exploit the constraints in the environment (i.e. the table) to bend the wrist in order to align the hand with the object and thus to achieve a more stable grasp [25] (Fig. 6). To bend the wrist the subjects seldom adopted the following strategy: they stiffened the elbow and slightly lowered the shoulder and trunk towards the table; this this proved more comfortable than extending the elbow. With the SW the only way to align hand and objects was by performing compensatory movements with the trunk and shoulder joints.

Some of these behaviors and differences are visible in the trajectories of the body segments and of the wrist angles of two representative tasks: jar lid opening and full jar transport (Fig. 4). In the jar lid task (Fig. 2b), the compliance of the wrist/orthosis could not provide any functional advantage, because the orthosis was not used for reaching and grasping the jar but actually for manipulating the lid (unscrewing it). Hence it was not surprising to find out that the SW and CW 
A
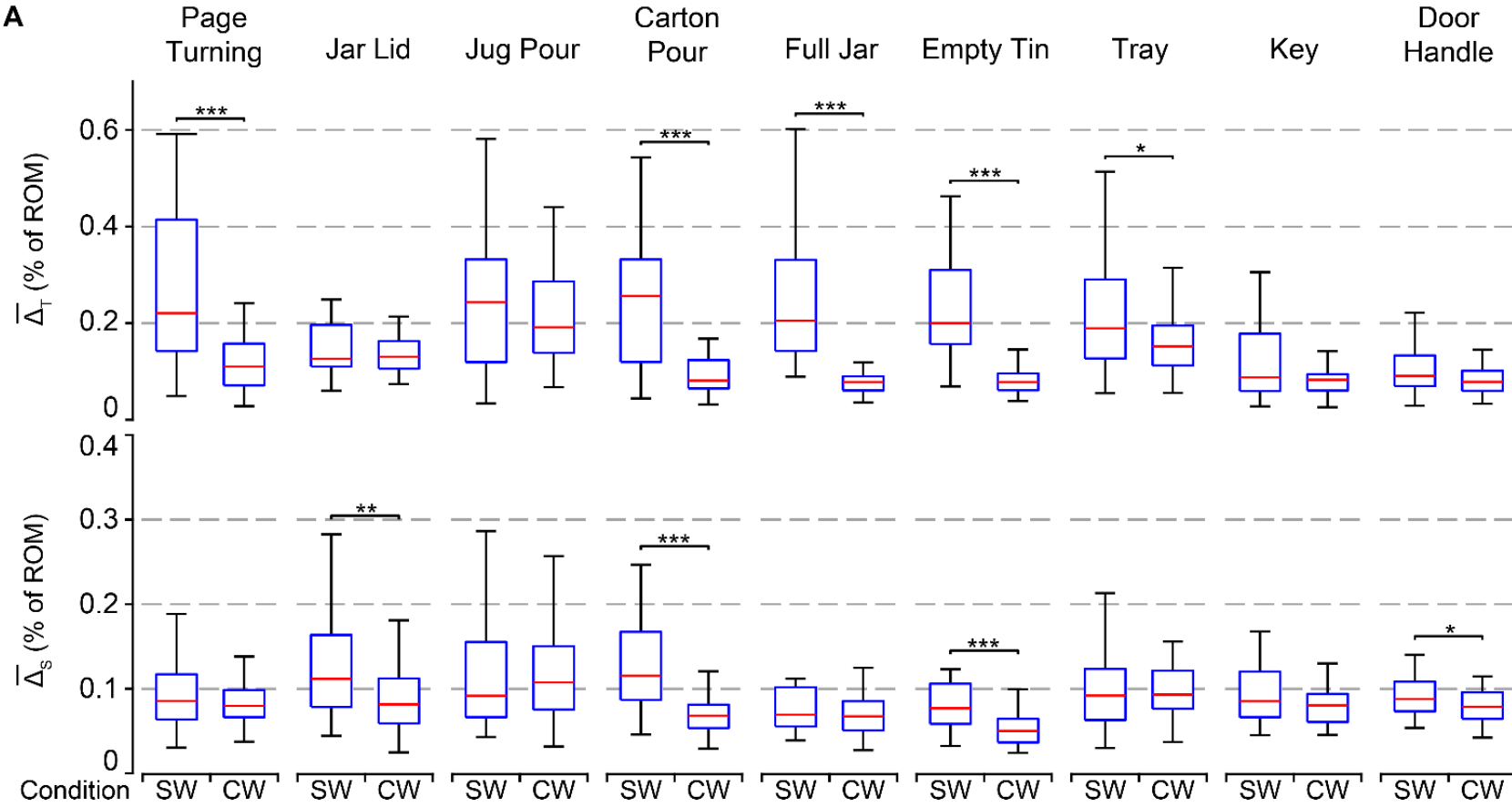

B

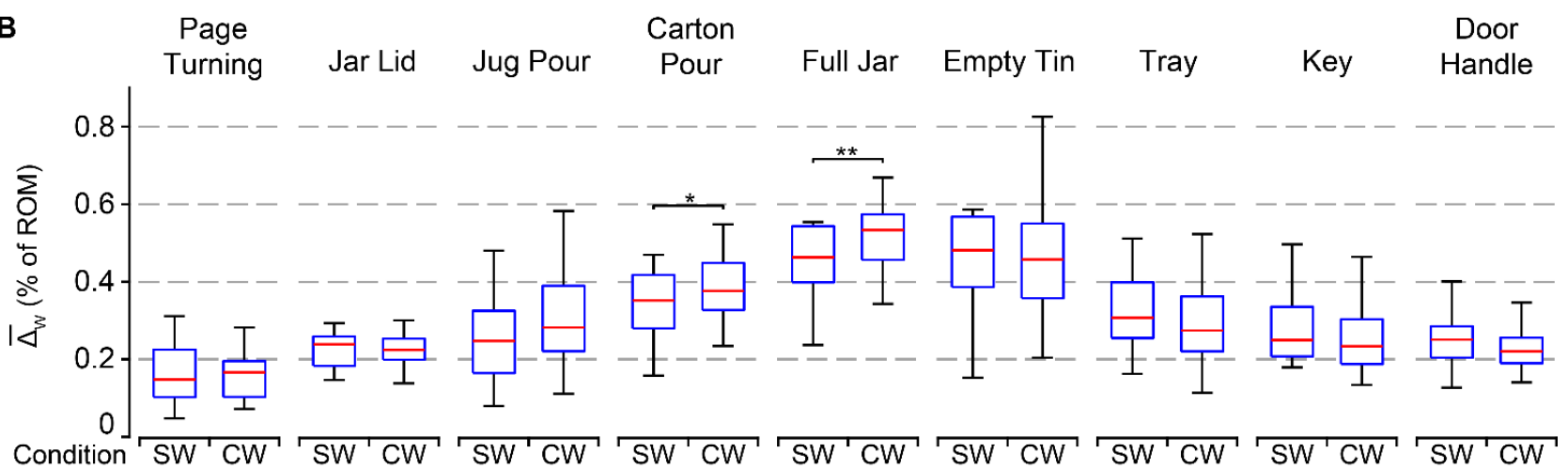

Fig. 5 Movement deviations during the 9 ADLs. A) Movement deviations of the proximal joints of the trunk $\left(\overline{\Delta_{T}}\right)$, and shoulder $\left(\overline{\Delta_{S}}\right)$ during the reaching and grasping phase. B) Movement deviations for the wrist $\left(\overline{\Delta_{W}}\right)$ during the holding and manipulation phase. Data from all subjects and repetitions for the stiff $(\mathrm{SW})$ and compliant $(\mathrm{CW})$ wrist configurations. Asterisks denote statistical differences between configurations as reported by the Wilcoxon ranked sum test $\left({ }^{*}=p<0.05 ;{ }^{* *}=p<0.01 ;{ }^{* *}=p<0.001\right)$.

conditions yielded to similar behaviors, as highlighted by the compensatory movements of the proximal joints (trunk and shoulder) during the RG phase (i.e. the reaching and grasping phase) (Fig. 4). Stiff and compliant wrists proved comparable also during the HM phase (i.e. the holding and manipulation phase) as displayed by the wrist angles. In the full jar transport task (Fig. 2e), the subject could instead exploit the compliance of the wrist as the orthosis was used for reaching for, grasping and lifting the jar from the table. In particular the subject could push the hand against the surface of the table in order to bend the wrist and align the envelope of the hand with the axis of the jar, prior to grasping it (akin to Fig. 6). As expected the functional advantages induced by the compliant wrist were nicely captured by the body angles that more closely matched the control condition with the $\mathrm{CW}$ (in particular the $\mathrm{T}_{\mathrm{F}}$ ), during the RG phase. Yet, during the HM phase, in which the subjects had to lift and transport the jar on the other side of the table, the $\mathrm{CW}$ yielded to oscillations of the wrist radial/ulnar deviation $\left(\mathrm{W}_{\mathrm{R} / \mathrm{U}}\right)$ and flexion/extension $\left(\mathrm{W}_{\mathrm{F} / \mathrm{E}}\right)$, due to the weight and inertia of the hand grasping the jar. Although very different, in both tasks the SW induced the subjects to compensate for the missing DoFs in the wrist by performing consistent compensatory movements during the RG phase; notably such deviations were maintained also during the following HM phase.

The average compensatory movements of the proximal joints (trunk and shoulder) proved generally lower with the $\mathrm{CW}$ than with the SW (Fig. 5A). This was statistically true for the trunk deviation, $\overline{\Delta_{T}}$, in five out of nine tasks: page turning $(\mathrm{p}<0.001)$, carton pouring $(\mathrm{p}<0.001)$, full jar $(\mathrm{p}<0.001)$, empty tin $(\mathrm{p}<0.001)$ and the tray $(\mathrm{p}=0.044)$ (Fig. 5A). Similarly, under $\mathrm{CW}$ the average glenohumeral joint angles, $\overline{\Delta_{S}}$, demonstrated lower than SW in four tasks: jar lid $(\mathrm{p}=0.002)$, carton pouring $(\mathrm{p}<0.001)$, empty tin $(\mathrm{p}<0.001)$ and door handle $(\mathrm{p}=0.026)$. The deviations of the wrist, $\overline{\Delta_{W}}$, during the HM phase demonstrated opposite trends; the SW demonstrated lower in two out of nine tasks: carton pouring $(\mathrm{p}=0.042)$ and full jar $(\mathrm{p}=0.003)$. No other statistical differences could be found between $\mathrm{CW}$ and SW (Fig. 5B). 


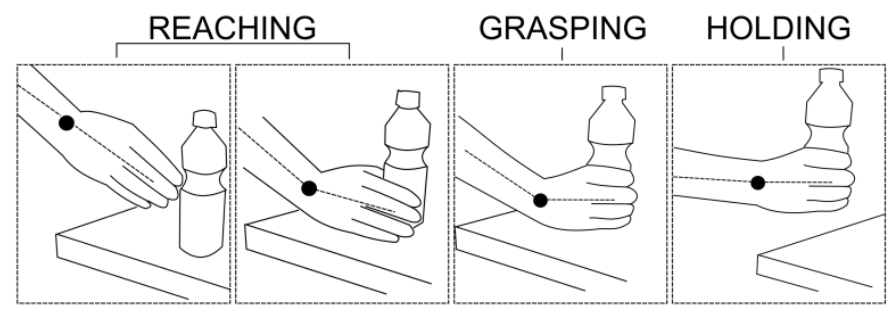

Fig. 6 Typical manipulation sequence when using the compliant wrist.

All in all these outcomes confirm our hypothesis that a transradial prosthesis would allow more natural operation if its wrist behaved compliant during the RG phase albeit they only partially confirm that a stiff wrist would be better during the HM phase. In fact the deviation analysis demonstrated that the compliant wrist outperformed - when not equaled - the stiff wrist in terms of significantly lower compensatory movements in the trunk and shoulder, during the RG phase in all analyzed tasks. During the manipulation phase, instead, the stiff wrist exhibited more natural movements (than the compliant one) mainly in those tasks involving the lifting of heavier objects (carton pouring and full jar transport). For the remaining tasks manipulating with a stiff or compliant wrist made no noticeable difference.

\section{DISCUSSION}

In order to contribute to the scientific debate on passive wrists for transradial myoelectric prostheses, in this work we searched for the most appropriate degree of compliance (either compliant or stiff) during the different phases of a motor task. Although gathered in simulated conditions with unimpaired subjects, the analysis on the body angles demonstrated that a compliant wrist induced lower compensatory movements than a stiff wrist, in the trunk and shoulder, during the preparation phase of the task, i.e. while reaching and grasping the target object. Nonetheless, during the actual manipulation phase, the stiff wrist exhibited more natural movements (than the compliant one), albeit only while handling heavier objects. Hence, our results support the hypothesis that a transradial prosthesis would allow easier and more natural operation if its wrist behaved compliant during the reaching and grasping phase, and stiff during the holding and manipulation phase.

This study was inspired by the work by Deijs and colleagues which assessed the compensatory movements in the shoulder of transradial amputees while wearing actual myoelectric prostheses endowed with stiff or compliant wrists [7]. In contrast to their hypothesis, to previous findings [5], [6], and perhaps to the general voice in the field, they could not reveal significantly different compensatory movements induced by different wrists, and suggested to search these differences in the trunk motions. Here by taking such suggestion we were able to demonstrate the different compensatory movements between wrists. However, this was not the only methodological difference with Deijs' and colleagues' work; other differences pertained to the chosen ADLs and to the metrics. They chose six tasks to explicitly assess shoulder movements while we chose nine from a standardized procedure (the SHAP); nonetheless the tasks largely overlapped from a functional perspective. In addition they measured the maximum deviations from the control condition while we assessed the average deviations. Finally we introduced the separation of the data in the three phases of the motor task. We argue that this was the key factor for identifying differences between $\mathrm{SW}$ and $\mathrm{CW}$ because it allowed to analyze in detail the compensatory movements throughout the phases (which are indeed very different). It also allowed to support the hypothesis that the trunk takes most of the compensatory action for aligning the prosthesis with the object, as suggested by Metzger et al. [20].

The ADLs were performed with the subjects standing in front of a table with the experimental materials. This precaution was taken to emphasize the required compensatory movements and thus to highlight differences between experimental conditions [7]. It should be noted indeed that the SHAP protocol explicitly targets the hand function and is not optimized for the wrist. In turn the wrist is rarely used in the SHAP and the subject sits and grasps the majority of the objects with the forearm/hand at the same height of the object, thus with limited need to orientate the wrist. Nonetheless we deemed important to test the ADLs of a standardized procedure, in order to allow for comparison, rather than making up new ones.

We assessed the compensatory movements in the shoulder $\left(\mathrm{G}_{\mathrm{A} / \mathrm{A}}\right.$ and $\left.\mathrm{G}_{\mathrm{F} / \mathrm{A}}\right)$ and trunk as these joints are known to be essential to compensate for the lost mobility of the arm in the case of a transradial amputation [7], [20], [27]. In particular we did not include the rotation of the shoulder as for limited workspaces (as those involved in this study) it proved highly correlated with the $\mathrm{G}_{\mathrm{A} / \mathrm{A}}$ [24]. Notably the compensatory movements of the proximal joints were analyzed during the reaching phase only, in agreement with previous literature [20], [22]. Indeed, as also shown in this work, the abnormal posture develops during the reaching phase, and does not vanish until the end of the motor task (Fig. 4). For example, the compensatory movements of the trunk induced by the stiff wrist during the reaching phase did not disappear during the manipulation phase.

The deviations of the wrist angles from the control condition become interesting during the holding and manipulation phase. Albeit the $\mathrm{CW}$ was generally preferable to a SW during RG, we found that for the HM phase the SW yielded to more natural (less bouncing) movements during the manipulation of heavy $(>525 \mathrm{~g})$ objects. This probably explains the preference for stiff wrists while handling heavy objects, as reported in previous studies [5]-[7]. Tasks involving fast or impulsive dynamics or heavy objects may become difficult to manage with compliant wrists as these may oscillate unexpectedly under proximal force actions. This is confirmed by Deijs' and collaborators' outcomes: amputees reported a "lack of stability" during tasks like riding a bike or the lifting of shopping bags; they also claimed that a compliant wrist could be "irritating and dangerous" as it could change position unintentionally while manipulating objects [7]. The claim finds support in the natural behavior of the human wrist, which tends to stiffen when the hand is subject to unstable loads [28], [29]. We argue that the lack of stability or the unnatural orientation of the compliant wrist may even induce the subject to adopt strategies to compensate for it [30], i.e. yielding to additional compensatory movements or muscle 
contractions in the proximal joints. Anecdotally, this was observed here when heavier objects were handled. These caused the $\mathrm{CW}$ to bend and therefore were transported with an unnatural orientation. This effect may not be negligible and may affect a wide range of ADLs in which the target object has to be maintained in a precise orientation during transport (e.g. vertical position of a jug of water).

A weakness of the present work is that individuals with amputations did not participate in the study. However, we were constrained by a lack of access to such individuals in the time frame available to us. So we chose to conduct this exploratory study by enrolling intact limbed subjects wearing an experimental orthosis. Earlier studies indicated that healthy subjects using orthoses exhibit mechanisms of compensation similar to prosthesis users, and that the magnitude of compensation could be even greater for the latter [16], [22]. In turn, we expect that if we replicated this work with upper limb amputees wearing actual prostheses, compensatory movements in the trunk would prove larger.

All in all our findings are of considerable importance in the domain of prosthetic design, as they indicate ways to improve the functionality of modern prosthetic wrists. Our results prove that the optimal compliance for a prosthetic wrist is specific to the phase of the motor task. They also suggest that a wrist with two degrees of compliance could significantly increase the functionality of the prosthesis, if automatically switched in synchronous with the onset of grasp, from compliant to stiff. The concept of such a wrist was presented earlier by our group [25] and this study clears the way towards the development of a miniaturized and clinically viable version of it.

\section{REFERENCES}

[1] M. Controzzi, C. Cipriani, and M. C. Carrozza, "Design of artificial hands: A review," in The Human Hand as an Inspiration for Robot Hand Development, vol. 95, R. Balasubramanian and V. J. Santos, Eds. Springer, Cham, 2014, pp. 219-246.

[2] R. Jacobs, "The addition of a flexible wrist unit to enhance prosthetic function," in MyoElectric Controls/Powered Prosthetics Symposium, 1993, pp. 63-67.

[3] F. Montagnani, M. Controzzi, and C. Cipriani, "Is it Finger or Wrist Dexterity That is Missing in Current Hand Prostheses?," IEEE Trans. Neural Syst. Rehabil. Eng., vol. 23, no. 4, pp. 600-609, 2015.

[4] H. H. Sears and J. Shaperman, "Electric Wrist Rotation in ProportionalControlled Systems," JPO J. Prosthetics Orthot., vol. 10, no. 4, pp. $92-$ 98, 1998

[5] P. J. Kyberd, "The influence of passive wrist joints on the functionality of prosthetic hands," Prosthet. Orthot. Int., vol. 36, no. 1, pp. 33-8, Mar. 2012.

[6] B. Petersen, "Preliminary feedback from field trial users of the motion control multi-flex wrist," in MyoElectric Controls/Powered Prosthetics Symposium, 2008, pp. 13-16.

[7] M. Deijs, R. M. Bongers, N. D. M. Ringeling-van Leusen, and C. K. van der Sluis, "Flexible and static wrist units in upper limb prosthesis users: functionality scores, user satisfaction and compensatory movements," $J$. Neuroeng. Rehabil., vol. 13, no. 1, p. 26, 2016.

[8] N. M. Bajaj, A. J. Spiers, and A. M. Dollar. "State of the art in prosthetic wrists: Commercial and research devices," in Proc. 2015 IEEE International Conference on Rehabilitation Robotics (ICORR), Singapore, 2015, p. 331-338.

[9] Ø. Stavdahl, "Optimal wrist prosthesis kinematics: three-dimensional rotation statistics and parameter estimation," Ph.D dissertation, Norwegian University of Science and Technology, Trondheim, Norway, 2002 .
[10] J. Davidson, "A survey of the satisfaction of upper limb amputees with their prostheses, their lifestyles, and their abilities," J. Hand Ther., vol. 15 , no. 1, pp. 62-70, 2002.

[11] A. Zinck, "The investigation of compensatory movements in prosthesis users and the design of a novel wrist," M.S. thesis, Dept. of Mechanical Engineering, University of New Brunswick, New Brunswick, Fredericton (Canada), 2008.

[12] P. S. Kidd, C. McCoy, and L. Steenbergen, "Repetitive strain injuries in youth," J. Am. Acad. Nurse Pract., vol. 12, no. 10, p. 413-426., Oct. 2000.

[13] L. E. Jones and J. H. Davidson, "Save that arm: a study of problems in the remaining arm of unilateral upper limb amputees," Prosthet. Orthot. Int., vol. 23, pp. 55-58, 1999.

[14] C. R. Gambrell, "Overuse syndrome and the unilateral upper limb amputee: Consequences and prevention," J. Prosthet. Orthot., vol. 20, no. 3, pp. 126-132, 2008.

[15] C. M. Light, P. H. Chappell, and P. J. Kyberd, "Establishing a standardized clinical assessment tool of pathologic and prosthetic hand function: Normative data, reliability, and validity," Arch. Phys. Med. Rehabil., vol. 83, no. 6, pp. 776-783, Jun. 2002.

[16] M. J. Major, R. L. Stine, C. W. Heckathorne, S. Fatone, and S. A. Gard, "Comparison of range-of-motion and variability in upper body movements between transradial prosthesis users and able-bodied controls when executing goal-oriented tasks," J. Neuroeng. Rehabil., vol. 11 , no. 1 , p. $132,2014$.

[17] T. Bertels, T. Schmalz, and E. Ludwigs, "Objectifying the Functional Advantages of Prosthetic Wrist Flexion," J. Prosthet. Orthot., vol. 21, no. 2, pp. 74-78, Apr. 2009.

[18] A. Murgia, P. Kyberd, and T. Barnhill, "The use of kinematic and parametric information to highlight lack of movement and compensation in the upper extremities during activities of daily living," Gait Posture, vol. 31, no. 3, pp. 300-306, 2010.

[19] A. H. Bottomley, "Myo-electric control of powered prostheses," J. Bone Joint Surg. Br., vol. 47, no. 3, pp. 411-5, Aug. 1965.

[20] A. J. Metzger, A. W. Dromerick, R. J. Holley, and P. S. Lum, "Characterization of compensatory trunk movements during prosthetic upper limb reaching tasks," Arch. Phys. Med. Rehabil., vol. 93, no. 11, pp. 2029-2034, 2012.

[21] S. L. Carey, R. V. Dubey, G. S. Bauer, and M. J. Highsmith, "Kinematic comparison of myoelectric and body powered prostheses while performing common activities," Prosthet. Orthot. Int., vol. 33, no. 2, pp. 179-186, 2009.

[22] S. L. Carey et al., "Compensatory movements of transradial prosthesis users during common tasks," Clin. Biomech., vol. 23, no. 9, pp. 1128$1135,2008$.

[23] A. G. Mell, B. L. Childress, and R. E. Hughes, "The effect of wearing a wrist splint on shoulder kinematics during object manipulation," Arch. Phys. Med. Rehabil., vol. 86, no. 8, pp. 1661-1664, 2005.

[24] F. Montagnani, M. Controzzi, and C. Cipriani, "Exploiting arm posture synergies in activities of daily living to control the wrist rotation in upper limb prostheses: A feasibility study," in Proceedings of the Annual International Conference of the IEEE Engineering in Medicine and Biology Society, EMBC, 2015, vol. 2015-Novem, pp. 2462-2465.

[25] F. Montagnani, M. Controzzi and C. Cipriani, "Preliminary design and development of a two degrees of freedom passive compliant prosthetic wrist with switchable stiffness," in Proc. 2013 IEEE International Conference on Robotics and Biomimetics (ROBIO), Shenzhen, 2013, pp. 310-315.

[26] I. A. Kapandji, The Physiology of the Joints: Upper Limb, Volume 1, 2nd ed., vol. 1. London: Churchill Livingstone, 1970.

[27] A. J. Metzger, A. W. Dromerick, C. N. Schabowsky, R. J. Holley, B. Monroe, and P. S. Lum, "Feedforward control strategies of subjects with transradial amputation in planar reaching," J. Rehabil. Res. Dev., vol. 47, no. 3, p. 201, 2010.

[28] N. Hogan, "Adaptive Control of Mechanical Impedance by Coactivation of Antagonist Muscles," IEEE Trans. Automat. Contr., vol. 29, no. 8, pp. 681-690, 1984.

[29] S. J. De Serres and T. E. Milner, "Wrist muscle activation patterns and stiffness associated with stable and unstable mechanical loads," Exp. Brain Res., vol. 86, no. 2, pp. 451-458, 1991.

[30] T. E. Milner and C. Cloutier, "Compensation for mechanically unstable loading in Voluntary Wrist Movement," Exp. Brain Res., vol. 94, no. 3, pp. 522-532, 1993. 